\title{
Presumptions in Communication
}

\author{
Andrei Moldovan \\ Universidad de Salamanca, Spain \\ e-mail: $\underline{\text { mandreius@usal.es }}$
}

\begin{abstract}
:
In the first part of this paper I consider the Gricean account of communication, as structured by the Cooperative Principle and the four maxims. Several authors, including Jean Goodwin [10], Fred Kauffeld [17], Michael Gilbert [7], Ernie Lepore and Mathew Stone [22], among others, argue that the Gricean view of communication fails in as much as it pretends to offer an account of all such human interactions. As Goodwin and Kauffeld suggest, a more promising starting point is to consider the variety of contextually determined presumptions that we make about speakers and that we rely upon in interpreting utterances. These presumptions are established in various ways, and are dropped, or defeated, in certain conditions. In order to clarify these aspects we need to inquiry into the nature of presumptions. I argue that Kauffeld's [18], [19], [20] account of presumptions is useful in this context. In the second part of the paper I look at what this account tells us about how, and in what conditions, presumptions in communication are rebutted.
\end{abstract}

Keywords: pragmatics, communication, cooperation, presumption, Grice.

\section{Introduction ${ }^{1}$}

In his seminal paper "Logic and Conversation", Paul H. Grice introduces the term 'implicature', as a name for a certain phenomenon he aims to characterize. He does not offer a definition of implicatures, but instead presents the phenomenon by appeal to examples. He later proposes five characteristics of conversational implicatures, one of which is calculability [15, p. 31]. Certain ingredients are essential in the calculation of the implicature: one is what is said by the utterance of the sentence that carries the implicature (its semantic content, or literal meaning), and another is the premise that "the hearer is entitled to assume" $[15$, p. 31$]$ that the speaker is observing the maxims, or at least the Cooperative Principle (CP, henceforth). As is well known, the $\mathrm{CP}$ reads as follows: "Make your conversational contribution such as is required, at the stage at which it occurs, by the accepted purpose or direction of the talk exchange in which you are engaged" [15, p. 27]. Starting from the CP, Grice proposes four maxims that govern conversational interactions and certain more specific submaxims, which, he writes, "will, in general, yield results in accordance with the Cooperative Principle" [15, p. 26]. These are the 
maxims of Quantity, Quality, Relation, and Manner. I skip a detailed presentation of these maxims, as they are well known to the reader.

Concerning the grounding of the $\mathrm{CP}$, Grice writes that he does not think of it as an empirical generalization. Grice also rejects the hypothesis (which he says he entertained at a certain moment) that the CP has a "quasi-contractual" basis, i.e., that it is based on some sort of implicit agreement among the participants in the conversation. Instead, he suggests that the $\mathrm{CP}$ and the maxims are grounded in the rationality of cooperative transactions:

I would like to be able to show that observance of the Cooperative Principle and maxims is reasonable (rational) along the following lines: that anyone who cares about the goals that are central to conversation/communication... must be expected to have an interest, given suitable circumstances, in participation in talk exchanges that will be profitable only on the assumption that they are conducted in general accordance with the Cooperative Principle and the maxims [15, pp. 29-30].

Grice does not say much about the relation between $\mathrm{CP}$ and the maxims. Although the maxims are not directly deducible from the $\mathrm{CP}$, it is clear from his writings that the latter is meant to ground them in some sense. As Eemeren at al. write, "the Cooperative Principle encompasses certain values (sincerity, efficiency, relevance, and clarity)" [6, p. 7], which correspond to Grice's maxims. ${ }^{2}$ Indeed, a person engaged in a cooperative activity seems to be moved by such values. Otherwise, it is questionable that we would be talking about a cooperative attitude at all. In calling the maxims "Quantity, Quality, Relation, and Manner", Grice says he is "[e]choing Kant" [15, p. 26]. This reference to Kant suggests that Gricean maxims are conditions that make communication a cooperative activity. That is, communication in which the four maxims are not followed cannot be cooperative. This interpretation is also suggested by Fred Kauffeld [17, p. 5], who writes that the relation between CP and maxims "is supported by a transcendental deduction of sorts based on the clear pragmatic importance of veracity, relevance, perspicuity, and economy to serious communication."

The Gricean view of communication has inspired an enormous literature and is central to the discussions in linguistics and philosophy of language on how to draw the semantics-pragmatics distinction. Some authors find Grice's CP unproblematic in its universality. For instance, Green writes: "Grice saw the relevance of the CP to language use as just the linguistic reflex of its general relevance. He saw it as governing all rational behaviour. If it does, we should certainly find it universally applicable with regard to language use, and I know of no genuine counterexamples" [12, p. 411]. However, it has been argued that there are genuine counterexamples. One line of criticism suggests that the four Gricean maxims are not sufficient to explain the wide variety of implicatures and related pragmatic phenomena. For instance, Geoffrey N. Leech argues that a further maxim concerning politeness needs to be added to the list, which he calls "the Tact Maxim" [21, p. 13]. In a simplified formulation this reads "Do not cause offence" (but a more complex formulation is required for it to be theoretically useful, see [21, pp. 109-112]). Leech argues that introducing such a maxim is necessary in order to explain the implicatures generated by polite requests such as "I wonder if you'd mind handing me that screwdriver?"

In a similar vein, Michael Gilbert argues that the Gricean maxims are

a necessary but not sufficient condition... Grice's maxims must be adapted to field and cultural norms and expectations. Gricean Maxims as expressed in his essay (Grice, [15]) are geared to British middle-class values and expectations... Other cultures and, possibly, fields require adjustment [7, p. 433]. 
He gives the example of cultures in which the maxim of Quality ("Do not say what you believe to be false.") is violated when it conflicts with other maxims. For instance, he argues that in the Mexican culture there is such a "Super-Duper maxim" which concerns politeness, and reads: "never be rude to a stranger". Not answering a request for information may be considered rude in this culture, so some information is always given upon request, even when the speaker has not good evidence that her answer is correct.

Now, Grice is aware that his CP is to a certain extent an idealization. He writes: "Our talk exchanges... are characteristically, to some degree at least, cooperative efforts" $[15$, p. 26, emphasis added]. But the problem is not only that the Gricean picture of communication is too idealized, i.e. that real life communication (and surely argumentation) does not seem to be that cooperative. Probably the most significant problem that it faces is that communication is sometimes not even supposed to be a cooperative activity. Jane Goodwin [10] makes this point forcefully with respect to courtroom debates. In the case of courtroom argumentative interactions, both the accusation but especially the accused are legitimately uncooperative and cannot be expected to cooperate. It is common that the accused has relevant information that she conceals. Goodwin discusses several Gricean maxims, and argues that there is no presumption that they are observed within the courtroom, due to the normative setting of the debate. For instance, Grice's maxim of Quality has a submaxim which reads: "Do not say what you believe to be false". But what this maxim requires is in sharp conflict with a maxim that is enforced in Anglo-American procedure for courtroom debate, which Goodwin calls 'Nonvouching':

Nonvouching: Presume that the advocate will not make explicit what she herself believes. [10, p. 269].

The judge immediately cautions a lawyer that fails to abide by this maxim. Another presumption that is reasonable to make in courtroom debates is the following:

Zealous Argument: Presume that the advocate will make the best case she can for her position. [10, p. 270].

The reason behind this maxim is the very nature of the job that advocates are required to do in such settings. This, again, conflicts with the first submaxim of Quality. The advocates are not required to form a careful and balanced judgement on whether the accused is guilty or not, but to build the best case they can for her or his culpability, and, respectively, to do their best to defend the accused. Presumptions such as these are relied upon in court in drawing conclusions, either in the form of implicatures, or in the form of explicit arguments.

In a similar vein, Kauffeld $[17$, p. 3] notes that there is a large class of human linguistic interactions that are "patently non-cooperative". Some such interactions, he writes, involve "a degree of coercion which precludes cooperation; in others one party is plainly indifferent to the objectives, goals, etc. of the other." In the first category he includes interrogations [17, p. 5], in which the information is extracted from a victim by means of threatening and torturing. Grice's PC reads that a cooperative participant in a conversation makes her contribution as is required "by the accepted purpose or direction of talk". But in this case there is no accepted purpose, as the victim is there against her will. The other category he mentions is that of institutional discourse, in which bureaucrats proceed on the basis of regulations and are indifferent to the goals and interests of those to which the discourse is addressed. Lepore and Stone [22, p. 221] mention such cases as well in arguing that communication is not a cooperative activity, not even in the minimal sense that the participants share the goal of mutual understanding. Usually, if mutual understanding is not reached the parties engaged in conversation make efforts towards it. But this is not always so. Lepore and Stone [22, p. 221] illustrate this point 
with the realistic dialogue in a comic strip due to Scott Adams, in which Alice, responsible for technical support, tells Tina the procedure to solve a computer problem:

Alice: Just disable the local cache mode to fix the MAPI settings and delete the duplicate messaging subsystem registry key.

Tina: What if I don't understand anything you just said right then?

Alice: Good grief! I can't make it any simpler.

Alice walks away commenting: "It's funny because it's cruel!" Cases of this kind show that mutual understanding is sometimes not a common goal of all participants in the conversation.

\section{Presumptions in Communication}

The discussion in the previous section suggests that not all communication is cooperative, not even in the minimal sense that the participants share the goal of mutual understanding. Of course, in many cases, communication is not competitive but collaborative. In those cases speakers might cooperate in a substantive sense, i.e., by subscribing to a common goal and contributing to its achievement. When communication is cooperative, speakers are presumed to observe the $\mathrm{CP}$, and in those cases the maxims do find grounding in the $\mathrm{CP}$. But the Gricean view of communication fails in as much as it pretends to offer an account of all human communicative interactions. The four maxims that Grice proposes are reasonable rules to follow only in certain kinds of communicative settings. In general, what we might presume about speakers and hearers vary in content depending on the normative setting on the conversation and many other contextual factors. In view of this variation, the natural suggestion to make is to give up the "Kantian" view of the maxims that speakers follow in communication. This is the conclusion that both Kauffeld [17, p. 14] and Goodwin [10, p. 275] suggest. Goodwin writes:

If, as I believe, such diversity of presumptions is the norm, then we should stop looking for foundational presumptions like the CP that will govern all talk exchanges. Instead, the vital question becomes: how do participants in a given talk exchange come to recognize the presumptions that are applicable to them? [10, p. 275].

The presumptions that we might reasonably make about speakers and hearers have a variety of sources. One source of presumptions is the normative setting of the conversation. As Goodwin [10] argues, courtroom debates are organized in such a way that presumptions concerning what advocates do are established from the very beginning. Kauffeld [17, p. 10], developing a suggestion in Stamp [24], identifies the source of the presumption of veracity in the intentional structure of the locutionary act of saying something, as outlined by Grice's [13], [14], famous analysis of speaker meaning. In the context of an interrogation there is no presumption that the victim will be sincere, as it is well known from the very beginning that she has no interest in being sincere. In casual conversations, jokes, etc., there is no presumption that Grice's maxim of Quantity is observed, as there is no exchange of information at all. In other cases, special norms of politeness specific to a culture must be considered, as Gilbert suggests. And so on.

This suggests a very different picture of communication, one that is more encompassing than the Gricean view. It is also a view of communication that gives a significant role to the notion of presumption. The idea is that in producing and interpreting utterances we rely on a series of contextually determined presumptions that function as useful tools for these purposes.

Now, while this approach to communication departs significantly from Grice's in the aspects mentioned, it does not depart so radically in emphasizing the role of presumptions in communication. 
Grice also mentions "presumptions" several times in his "Logic and Conversation". For instance, in his account of the derivation of implicatures he writes that the speaker "is to be presumed to be observing the conversational maxims, or at least the Cooperative Principle" [14, p. 30]. However, he does not clarify what a presumption is, and does not mention any interesting consequence that results from treating the claim that the speaker observes the maxims as a presumption, as opposed to an assumption, or a belief.

\section{Conditions on an Account of Presumptions in Communication}

In the rest of this essay I address the question concerning what presumptions are. There are various accounts of presumptions available in the literature, but not all of them fit best the view of communication that I have outlined so far. As a starting point, notice that an account of presumptions that plays a role in interpreting linguistic utterances must fulfil certain conditions. I list below the three conditions and then I go on to discuss them separately:

1. We need a notion of presumption that captures an ordinary practice.

2. The kind of presumption we are interested in is a propositional attitude, or maybe a kind of inference, but not a speech act.

3. Presumptions in communication are rebuttable or defeasible.

Condition (1) states that we need a notion of presumption that captures an ordinary practice. Indeed, a technical and partly stipulative notion of presumption cannot do the job that it is put to in the Gricean account of implicature calculation. Grice characterizes the inference that leads to the implicatum as one that speakers and hearers must be, at least in principle, able to make. This is not a theoretical derivation, or else the account would say little about how speakers manage to arrive at implicatures. Of course, this derivation is not meant to be psychologically real either, as speakers and hearers rarely, if ever, go through all the steps of the inference explicitly. As Kent Bach notes, "When he illustrated the ingredients involved in recognizing an implicature, [Grice] was enumerating the sorts of information that a hearer needs to take into account, at least intuitively, and exhibiting how this information is logically organized" [3, p. 8, emphasis added]. Implicatures are captured intuitively, but in those cases in which the implicature is present, the intuition is replaceable by an argument that leads to the conclusion that the speaker intends to convey the implicatum. As, Grice writes, "unless the intuition is replaceable by an argument, the implicature (if present at all) will not count as a conversational implicature" [15, p. 31]. So, the information that the speaker is presumed to be truthful, relevant etc. is what hearers use, explicitly or implicitly, when deriving the implicature. For that reason the notion of presumption relevant here cannot be a theoretical one, but a notion available to the speaker. Those accounts that introduce a technical notion of presumption, rather than aiming to analyse the ordinary notion of presumption in natural language, will not be fit for the present purpose ${ }^{4}$.

Condition (2) is that the notion of presumption that we need has to be one that picks out a certain propositional attitude, or maybe a kind of inference, but not a speech act. Probably the most well known account of presumption as a speech act is due to Walton [26]. He characterizes the speech act of presumption in relation to the allocation of the burden of proof in an argumentative dialogue. The key feature of presumptions, according to Walton [26] is that they reverse the burden of proof in an argumentative interaction. This "analysis of presumption" takes it to be "a kind of speech act that is half way between assertion and (mere) assumption" [26, p. 138]. That is because an assertion normally carries a burden of proof, while assumptions "are freely undertaken and can freely be rejected in a dialogue." In contrast with these speech acts, "when a presumption is brought forward by a proponent, the burden is on the respondent to refute it, or otherwise it goes into place as a commitment" [26, $p$. 
138]. More recently Bermejo-Luque proposed a somewhat similar analysis of the speech act of presumption: "I propose the following definition: a presumption is the speech-act of putting forward a proposition as a reasonable assumption." $[4, \mathrm{p} .4]^{5}$

However, I am not convinced that presumptions are fundamentally speech acts, nor that there is a speech act of presuming at all. In order to shed some light on this issue, it is useful to look at Austin's [2, p. 79] characterization of explicit performatives, i.e., verbs or expressions the purpose of which is to make explicit what performative act is being realized. Austin notes that there is a significant difference between, on the one hand, expressions such as 'I thank' and 'I apologize', which are explicit performatives, and, on the other hand, 'I feel grateful' and 'I repent', which are used to report on or "exhibit" [2, p. 83] certain feelings or attitudes. He notes that there is a third category of "not pure but half descriptive" (and half performative) verbs, such as 'I am grateful' and 'I am sorry'. These verbs suffer from a certain "ambivalence", as they can be used either to make a speech act of thanking or apologizing, or to merely exhibit one's attitude or feeling. Austin [2, p. 79f] proposes four tests in order to distinguish the performative use from the exhibitive use of these ambivalent expressions. I mention in what follows only the first two tests:

One test would be whether it makes sense to say 'Does he really?' For example, when someone says 'I welcome you' or 'I bid you welcome', we may say 'I wonder if he really did welcome him?' though we could not say in the same way 'I wonder whether he really does bid him welcome?' Another test would be whether one could really be doing it without actually saying anything, for example in the case of being sorry as distinct from apologizing, in being grateful as distinct from thanking, in blaming as distinct from censuring' [2, p. 79-80].

Austin argues that expressions such as 'I argue', 'I conclude', 'I testify', 'I admit' and 'I concede' ${ }^{6}$ appear to be pure performatives, and to pass the tests successfully. However, he adds that 'I assume that...', 'I suppose that...' and 'I presume that...' do not successfully pass the tests. Indeed, at least the two tests mentioned above clearly suggest the use of these verbs is not performative. It does make sense to ask during a trial, when we see the prosecutor treat the accused as guilty, "Does the prosecutor really presume the accused is innocent?". Second, one can presume something without saying anything. It seems uncontroversial that we can presume, assume or suppose something without saying anything. This is not the case for many standard speech act verbs, such as 'assert' or 'promise'. It is difficult to see how one could promise something or assert something without uttering a sentence, or at least perform an act that is endowed with meaning. Austin concludes that 'I assume', 'I suppose' and 'I presume' "operate in the ambivalent way that 'I am sorry for...' operates: this sometimes is equivalent to 'I apologize', sometimes describes my feelings, sometimes does both at once" [2, p. 87].

In fact, it seems to me the sense of 'I assume', 'I suppose' and 'I presume' in which they refer to a cognitive attitude is the fundamental. Consider again Austin's second test mentioned above. An assumption, supposition or presumption is not necessarily established or formed in the moment in which one uses the corresponding expressions, or by uttering them. Instead they are commonly used to express one's attitude towards a particular proposition, that is, in a purely descriptive way. I might suppose that the car is where I left it this morning, and act on this supposition, without telling you anything about what I suppose. I might presume that you brought back the book to the library and not say anything in doing so. In this sense they are similar to 'I doubt that', 'I believe that' or 'I am convinced that', which do not seem to have a speech act use at all. This is a prima facie reason to take 'I presume' to refer primarily to a cognitive act or state, and only derivatively to a kind of speech act.

Finally, condition (3) requires that presumptions in communication be rebuttable or defeasible. An account of presumptions in communication must capture the way these presumptions are treated. 
Independently of their source (e.g., the normative setting of courtroom debates, etc.), presumptions in communication are defeasible, in the sense that they might be dropped in face of contrary evidence. Sometimes hearers discover that speakers do not act sincerely, perspicuously, or say things that are not relevant to what is at issue. Consider, for instance, the norm that lawyers are presumed to observe, which requires that they make the best case they can for their position (i.e., what Goodwin [10, p. 270] calls Zealous Argument). But suppose we encounter evidence that a particular lawyer has been bribed to build a weaker case for her position than she could. In that scenario it is reasonable to drop the Zealous Argument presumption. After all, this is why they are characterized as presumptions: because they can be rebutted.

To take a further example, consider Grice's [15, p. 32] example of the gas station discussion, which goes as follows:
A: I am out of petrol.
$\mathrm{B}$ : There is a garage round the corner.

Intuitively, in the right context, B's utterance carries the implicature that the garage is, or at least might be, open, sells petrol, etc. According to Grice [15, p. 32], this implicature is generated by the presumption that the speaker is observing the maxim of relevance. But if we find out that B is a child, or someone completely unfamiliar with cars, we might doubt that B has good evidence for her claim (as the second submaxim of Quality requires), or that she has considered the opening hours of the station (as the maxim of Relation requires). In these cases we are not warranted to draw the implicature that the gas station is open. Such presumptions in everyday conversation are systematically cancelled or dropped in view of contrary evidence. An account of presumptions in communication must take into consideration this aspect of presumptions.

\section{Kauffeld's Account of Presumptions and the Presumptive Inference}

One account of presumptions that looks promising in view of the three requirements discussed above is due to Fred Kauffeld [18], [19], [20]. The aim of this proposal is to capture the notion of presumption in its everyday use, and to understand our ordinary practice of presuming and using presumptions in reasoning and communication. It is not part of a process of theory building, and does not aim to establish a normative model of presumption. Thus, Kauffeld's notion of 'presumption' is not to be understood as a term of art, or a theoretical concept. He writes:

This attempt at analysis and explication ... is methodologically situated in traditions of "ordinary language philosophy," and it leans heavily on the pioneering work of J. L. Austin, G. J. Warnock, the non-theoretical aspects of H. P. Grice's analysis of utterancemeaning, Dennis Stampe's studies of speech acts and so on [19, p. 1].

On Kauffeld's [18, p. 135] analysis of ordinary presumptions, a presumption is a conclusion established by a certain kind of inference. Kauffeld [18, p. 136] explicitly rejects the identification of presumptions with mechanisms for reallocating the burden of proof, pointing out that there other mechanisms that have the same effect. The fact that presumptions put forward in an argumentative dialogue have the effect they have on the distribution of the burden of proof is explained in Kauffeld [18, pp. 143-144] and [19] on the basis of the form of the inference and the grounds from which the inference is drawn.

According to this account, the presumptive inference is not identified by its form, but rather by the content of its premises: "To presume that $p$, in the ordinary sense of the term is to infer that $p$ on the 
supposition some agent has made, is making, or will make it the case that $p$, rather than risk criticism, retribution, etc. for failing to do so.” [18, p. 140] Later Kauffeld expends on this characterization:

On the supposition that a morally motivated agent would not act in a way that would provide others with reasonable (and perhaps unanswerable) basis for objection, criticism, resentment, reprobation, etc., those others may reasonably infer that in this particular case the agent is acting responsibly, truthfully, dutifully, and so on. We ordinarily identify such suppositions based on an agent's commitments as "presumptions" [19, p. 3].

Kauffeld [20,p. 5] notes that presumptions, on his view, have three "components", which are the following:

I. "the supposition that some agent (Ag) has obligation or other commitment owing to some other agent(s) and/or to herself that Ag is to do x";

II. "the supposition that, Ag has made, is making, or will make it the case that Ag has done $\mathrm{x}$, rather than risk resentment, retribution, etc. for failing to do $\mathrm{x}$;"

III. "the inferred conclusion that Ag has done, is doing, or will do x."

Kauffeld [20] indicates that the presumption is the conclusion of an inference, but does not put forward (I) and (II) as its premises. However, it is possible to reconstruct the inference in this way. The result would be the following (call it K1; I simplify the times of the verb):

i. Ag has an obligation or other commitment to do $\mathrm{x}$.

ii. Ag will do $\mathrm{x}$ rather than risk resentment for not doing $\mathrm{x}$.

Therefore,

iii. Ag will do $\mathrm{x}$.

The question now is whether this is a charitable reconstruction of Kauffeld's account of the presumptive inference. Derek Allen [1, p. 2] suggests it is not, as he finds the inference to be circular: (ii) already entails (iii), that $A g$ will do $x$. Allen writes that, apart from stating (iii), (ii) only adds an explanation of why (iii) is the case, which is what the part introduced by "rather than" conveys. This explanation, Allen [1, p. 1] suggests, could be unpacked as follows:

(E) A will do $\mathrm{x}$ because A would rather do $\mathrm{x}$ than risk resentment for not doing $\mathrm{x}$.

Moreover, whatever role (i) plays it relates to what comes after "rather than" in (ii), so it does not support the claim that Ag will do x. The role that (i) might play is either to give a reason for the claim that A risks resentment or regret for not doing $\mathrm{x}$, or to offer an explanation of this fact. In any case, (i) does not add any reason to believe (iii). This means that (iii) is offered no support by the above inference. If this is so then this inference is indeed a case of petitio principi, or vicious circularity. As a result, this is not a charitable reconstruction of Kauffeld's proposal.

However, there is an option we have ignored: (E) might be very well understood as an argument, and not as an explanation. Interpreting it in this way would be more charitable, as it would avoid the vicious circularity. Eventually, Allen [1, p. 2] proposes a reconstruction along these lines. This is the following (call it K2):

i. Ag has an obligation or other commitment to do $\mathrm{x}$. Therefore presumably, 
ii. Ag has done, is doing, or will do $\mathrm{x}$.

Allen adds: "I would then say that if [(i)] is true, its truth counts in favour of the truth of [(ii)], and so I would say that [(i)] is positively relevant to [(ii)]. Further, I would be justified in saying this, assuming that I am justified in believing (R)" $[1, \text { p. } 2]^{7}$. Here, $(\mathrm{R})$ is the following claim:

(R) A would rather do $\mathrm{x}$ than risk resentment for not doing $\mathrm{x}$.

Now, I am not sure why Allen does not add (R) as a premise of the presumptive inference. I suppose that he aims to capture the inferential step as one of a special kind, not reducible to a deductive or inductive inference. ${ }^{8}$ However, I do not find it more perspicuous or closer to Kauffeld's intentions to reconstruct the inferential step as being of a special kind (one that is warranted by $(\mathrm{R})$ ) as opposed to treating $(\mathrm{R})$ as a premise. To the effect of assessing the inference, the option of taking $(\mathrm{R})$ to be a premise or taking it to be a warrant of the inferential step seems to make no significant difference. In both cases, if we are not warranted to accept (R), we are not justified accepting the presumption. Moreover, Allen's reconstruction (K2) of the presumptive inference analyses the notion of presumption in terms of what we might presumably infer, but leaves the latter notion unanalysed. As a result, one is left wondering what 'presumably' might mean. In turn, Kauffeld's account, which Allen interprets, does not seem to be affected by this kind of conceptual circularity.

In view of these considerations, I think it would be more perspicuous to eliminate the qualifier 'presumably', and take (R) to be a premise of the argument. Moreover, I propose to reformulate (R) as (R'), which, I think, captures better the underlying idea:

(R') If A risks regret or resentment for not doing $\mathrm{x}$, then $\mathrm{A}$ will do $\mathrm{x}$.

I propose to reconstruct Kauffeld's presumptive inference as follows (call it K3):

i. Ag has an obligation or other commitment to do $\mathrm{x}$.

ii. If $\mathrm{Ag}$ has obligation or other commitment to do $\mathrm{x}, \mathrm{Ag}$ risks regret or resentment for not doing $x$.

iii. (R') If Ag risks regret or resentment for not doing $\mathrm{x}$, then $\mathrm{Ag}$ will do $\mathrm{x}$.

Therefore,

iv. Ag will do x.

Reconstructed in this way, premise (i) corresponds to Kauffeld's supposition (I), and (iii) (which is (R')), to Kauffeld's supposition (II). I have also added premise (ii). This premise makes explicit the relation between the existence of A's commitment or obligation to do $\mathrm{x}$, on the one hand, and the existence of the risk of resentment or regret that A faces. The relation is straightforward and unproblematic, and making it explicit helps exhibit the structure the reasoning behind the conclusion (iv).

\section{Presumptions are Rebuttable}

Kauffeld's account of presumption is particularly fit for the kind of presumptions in communication we aim to characterize. The account grounds presumptions in the motivation that agents have for abiding by norms and fulfilling their obligations, motivation which, in turn, is grounded in the risk of regret and resentment that those who fail to live up to their obligations or commitments face. This seems to fit the structure of reasoning behind our endorsement of the claim that a speaker is, for instance, sincere. One 
reason why we presume speakers are sincere is that they would otherwise risk resentment and/or risk regretting their insincerity. Although this reasoning does not apply universally, it does seem to apply to cases in which we do make such presumptions in communication. And the same could be said of the presumption corresponding to Grice's other maxims. Our main motivation for providing all the information required, as the maxim of Quantity indicates, is that we want to avoid being criticized for having a non-cooperative attitude. Consider also communicative interactions that are non-cooperative. As Goodwin [10, p. 270] suggests, the following presumption is typical of courtroom debates:

Zealous Argument. Presume that the advocate will make the best case she can for her position. What is the reason for making such a presumption? One good reason is that otherwise the advocate would face resentment from the authorities that gave her the responsibility of building the case. The structure of the reasoning that leads to this presumption seems to be well reflected by Kauffeld's analysis.

I have suggested above three conditions that an account of presumptions in communication must fulfil: (1) that the notion of 'presumption' we are interested in is that of an ordinary practice; (2) that a presumption in communication is an inferentially grounded propositional attitude and not a speech act; that (3) that presumptions in communication are rebuttable or defeasible. Kauffeld's account of presumptions fulfils the first two conditions: it aims to characterize our everyday practice of making presumptions, which, he argues, are not speech acts, but propositions inferred from premises of a certain kind. I do not discuss here the question whether Kauffeld's account offers a correct analysis of the ordinary practice of presuming or not. ${ }^{9}$ But notice that if the analysis turns out to characterize only a subclass of presumptions, and not the entire category, this would not prove especially problematic in relation to conditions (1) and (2), as long as the analysis does capture a real ordinary practice.

Let us now consider condition (3), that presumptions are rebuttable, and see whether ordinary presumptions as characterised by Kauffeld's account fulfil it. To begin with, notice that (K3) is a valid inference. If premises (i), (ii) and (iii) obtain, then the conclusion (iv) also obtains as a matter of logical consequence. This means that this is a monotonic inference, and so a non-defeasible one. Godden and Walton [8] and Godden [9] do not reconstruct Kauffeld's account of the presumptive inference as I have done above, but still see this as a "problem" for the account. The problem is that "presumptions do not seem to retain the property of defeasibility" [8, p. 323]. In order to illustrate this point they present the much-discussed example of the sleepy soldier. Here is the example:

Consider the case where it is a soldier's duty to raise the flag at dawn, but he is very unreliable and tends to sleep in. Consider now our presumption (as Kauffeld would have us talk of it) that $p$ : the soldier will raise the flag at dawn. In one sense, the presumption that $p$ does not disappear in the face of evidence that the social bonds obliging the soldier to bring it about that $p$ will not be met [8, p. 323].

The idea is that on Kauffeld's account, "the predictive force of the expectation is grounded in its normative force," and since the "force" of the norm is not affected by the soldier's conduct, we are entitled to the presumption that the soldier will raise the flag, no matter what additional information we might obtain about his conduct. ${ }^{10}$ The objection is that, on Kauffeld's account, the presumption that Ag fulfils her duty and acts in accordance with her normative commitments seem to stand unaffected in face of any contrary evidence, as long as the normative basis of the duty is unaffected (i.e., as long as she is still under the obligation to do it). This is, potentially, a serious problem for the account. It cannot be correct to say that we are still entitled to presume that the soldier raised the flag, as long as the normative grounds are unaffected, even if we found out he did not raise the flag. Otherwise, we would be entitled to presume that any person accused of any crime or misconduct and proven guilty of this is innocent. 
In discussing the case the sleepy soldier, Kauffeld [19, p. 2] notes that this criticism does not affect his account per se. The account aims to characterize the ordinary notion of 'presumption' and out everyday practice of presuming. If it turns out that our ordinary practice of presumptions does not have certain desirable properties, such as defeasibility, then probably they are not that useful for Godden and Walton's theoretical purposes, which are those of characterizing correct argumentation. ${ }^{11}$ But Kauffeld [19] seems to admit Godden and Walton's main point. He comments that, indeed,

we might continue to presume that Smith [the sleepy soldier] will raise the flag and are entitled to that presumption, even though we may have observationally based knowledge warranting belief that Smith will not. We would not regard the proposition presumed as a reliable prediction $[19$, p. 6$]$.

He adds: "We are commonly justified in accepting propositions regarding human acts which are from a predictive point of view false;" [19, p. 7]. My reaction is here the one Harvey Siegel also expressed: "But once we know that [the agent] is unlikely to do something, how can we be entitled nevertheless to expect him to do it anyway, given the negative [evidence]?" [23, p. 4]. Are we willing to presume (in the ordinary sense) that Smith has raised the flag at dawn even when the time for him to do it has passed and we know he did not do it? Although my intuitions about the use of the word in ordinary contexts are not strong, I am inclined to answer that we are not.

Despite Kauffeld's comments quoted above, I maintain that presumptions are rebuttable in the face of contrary evidence. Consider a situation in which the flags were stolen, or one in which Smith never received the order to raise the flag. ${ }^{12}$ In those cases the supposition (I) needs to be dropped: that is, Smith has no obligation to raise the flag, as one has no obligation to do what one cannot do. Now consider a situation in which we know the order was conveyed, the flags are where they are supposed to be, and we know Smith has committed himself to raising the flag. Still, when dawn breaks, Smith deliberately refuses to raise the flag, and expresses his intention to stay in bed until noon. Do we go on to presume that he will raise, and later on, that he did raise it, although we know he did not do it? On Kauffeld's account, reconstructed above as K3, what fails in this case seems to be premise (iii), which reads: if Ag risks regret or resentment for not doing $x$, then $A g$ will $d o x$. This is the content of Kauffeld's supposition (II). Are we entitled to suppose that $p$ is the case even if we know p is false? Intuitively, we are not. This supposition must be dropped if we know that the conditional is false for a given agent at a given time. Indeed, sometimes agents prefer risking resentment or regret to fulfilling their duties.

What this means is that Godden and Walton's criticism fails. Kauffeld's account does not predict that "the presumption that $p$ does not disappear in the face of evidence that the social bonds obliging the soldier to bring it about that $p$ will not be met" $\left[8\right.$, p. 323]. ${ }^{13}$ The presumption does disappear in case we are not warranted anymore to the supposition (II). And this is the case when we have strong evidence that the risk of resentment and regret is insufficient to motivate Ag to act as her obligations or commitments require of her. How strong must this evidence be? And, to what extent must her commitment-based motivation be undermined? These are difficult questions that I do not know how to answer. Maybe we are entitled to certain suppositions of the form (I) and (II) in spite of some contrary evidence. Kauffeld presents a case in which we have some inductive evidence that the corresponding premise (iii) does not obtain and still we are warranted to suppose it, and so to infer the conclusion of the presumptive inference. He writes: "A teacher, for example, might say to her class, "Your papers are due tomorrow, and I presume that you will all have them in on time," knowing full well that several members of the class routinely turn their papers in late" [19, p. 7].

Finally, notice the way in which Godden and Walton's criticism is framed: the problem for Kauffeld's account is, allegedly, that "presumptions do not seem to retain the property of defeasibility". 
That is, presumptions should be defeasible inferences and on this account they are not. Indeed, presumptions, on the reconstruction of Kauffeld's account of them that I proposed above (i.e., K3), are not, strictly speaking, defeasible, as the presumptive inference is monotonic. But an account of presumptions might predict that they are not defeasible inferences and still make correct predictions about for problematic cases such as that of the sleepy soldier. On my reconstruction K3 of Kauffeld's account, presumptive inferences are not defeasible (i.e., non-monotonic), but they are rebuttable. The conclusion cannot be retained when one of the suppositions on the basis of which it is drawn fails to be warranted.

\section{Conclusion}

That presumptions are rebuttable is a significant point to the project of reconsidering the Gricean view of communication. As I pointed out above, presumptions in communication are rebutted when contrary evidence is available. In those cases we simply do not infer the same implicatures that we infer when we do presume the speaker is sincere, relevant, is building the best case she can for a particular claim etc. Of course, many other questions need to be addressed in relation to the role presumptions of the kind discussed here play in communication. One such question concerns the strength of the reasons needed in order to cancel the supposition (I) and (II) that, on Kauffeld's account, ground the presumptive inference. I have not addressed this difficult question in the present paper. In turn, what I hope to have shown is that Kauffeld's account of presumptions is a very adequate tool for pursuing the project that Goodwin and Kauffeld, among others, advance, that of extending and correcting the Gricean view of communication. In particular I have argued that presumptions, on this account, are rebuttable in the right way.

\section{References}

1. Allen, D. Commentary on: Fred J. Kauffeld's "The epistemic relevance of social considerations in ordinary day-to-day presumptions". OSSA Conference Archive, Paper 88, 2013.

2. Austin, J. L. How to do Things with Words: The William James Lectures' delivered at Harvard University in 1955. In. J. O. Urmson (ed.), Clarendon: Oxford, 1962.

3. Bach, K. The Top 10 Misconceptions about Implicature. In. B. Birner, G. Ward (eds.), A Festschrift for Larry Horn, John Benjamins: Amsterdam, 2005.

4. Bermejo-Luque, L. Assessing presumptions in argumentation: Being a sound presumption vs. being presumably the case. OSSA Conference Archive, Paper 22, 2013.

5. Eemeren, F. H., van, Grootendorst, R. Speech Acts in Argumentative Discussions. Foris Publications: Dordrecht-Holland/Cinnaminson-U.S.A, 1984.

6. Eemeren, F. H., van, Grootendorst, R., Jackson, S., Jacobs, S. Reconstructing argumentative discourse. U. Alabama Press: Tuscaloosa, 1993.

7. Gilbert, M. Familiars: Culture, Grice and Super-Duper Maxims. In. D. Mohammed, M. Lewiński (eds.), Argumentation and Reasoned Action: Proceedings of the 1st European Conference on Argumentation, Lisbon, 2015, vol. 2, College Publications: London, 2016, pp. 431-438.

8. Godden, D., Walton, D. A theory of presumption for everyday argumentation. Pragmatics and Cognition, 15 (2), 2007, pp. 313-346.

9. Godden, D. Presumptions in argument: Epistemic versus social approaches. In. F. Zenker (ed.), Argumentation: Cognition \& Community. Proceedings of the 9th Biennial Conference of the Ontario Society for the Study of Argumentation (OSSA), 18-21 May 2011, CD-ROM. Windsor, ON: OSSA, 2011, pp. 1-13. 
10. Goodwin, J. The noncooperative pragmatics of arguing. In. Pragmatics in 2000: Selected papers from the 7th International Pragmatics Conference, vol. 2, 2001, pp. 263-277.

11. Govier, T. A Practical Study of Argument. Wadsworth: Belmont, 2010.

12. Green, G. M. The universality of Gricean interpretation. Proceedings of the Berkeley Linguistics Society, 16, 1990, pp. 411-428.

13. Grice, H. P. Meaning. The Philosophical Review, 66, reprinted in Grice 1989, (1957), pp. 377-88.

14. Grice, H. P. Utterer's meaning and intention. Philosophical Review, 78 (2), 1969, pp. 147-177.

15. Grice, H. P. Studies in the Way of Words. Harvard University Press: Cambridge, Harvard, 1989.

16. Hansen, H. Theories of Presumptions and Burdens of Proof. OSSA Conference Archive, 2003.

17. Kauffeld, F. J. Grice without the Cooperative Principle. OSSA Conference Archive, 2001.

18. Kauffeld, F. J. The ordinary practice of presuming and presumption with special attention to veracity and the burden of proof. In. F. H. v. Eemeren, J. A. Blair, C. A. Williard, A. F. Snoeck Henkemans (eds.), Anyone Who has a View: Theoretical Contributions to the Study of Argumentation, Kluwer Academic Publications: Dordrecht, 2003, pp.132-146.

19. Kauffeld, F. J. Presuming and presumption in everyday argumentation: A response to Godden and Walton. In. M. Guarini, S. Pender (eds.), Argument Cultures: Proceedings of the Eighth OSSA Conference, Ontario Society for the Study of Argument: Windsor, 2009.

20. Kauffeld, F. J. The epistemic relevance of social considerations in ordinary day-to-day presumptions. OSSA Conference Archive, Paper 87, 2013.

21. Leech, G. N. Explorations in Semantics and Pragmatics. Benjamins, 1980.

22. Lepore, E., Stone, M. Imagination and Convention: Distinguishing Grammar and Inference in Language. Oxford University Press: Oxford, 2015.

23. Siegel, H. Presumptions in argument: Epistemic versus social approaches. OSSA Conference Archive, 2011.

24. Stampe, D. On the Acoustic Behavior of Rational Animals. University of Wisconsin: Madison, 1967.

25. Ullman-Margalit, E. On presumption. Journal of Philosophy, 80 (3), 1983, pp.143-163.

26. Walton, D. N. The speech act of presumption. Pragmatics and Cognition, 1 (1), 1993, pp.125-148.

\section{Notes}

1. A previous version of this paper was delivered at the conference "Presumptions, Presumptive Inferences and Burden of Proof" (April 26-28, 2016 University of Granada, Spain). I would like to thank all the participants at this conference, especially David Godden, Fred Kauffeld and Marcin Lewinski, for their valuable comments.

2. See also van Eemeren and Grootendorst [5, p. 121]: the idea that argumentation is a cooperative activity the purpose of which is to resolve disputes about expressed opinions is central to pragma-dialectics' view of argumentation.

3. I am grateful to Lilian Bermejo-Luque for this reference.

4. Ullman-Margalit writes that when it comes to presumptions, "[e]xplication is usually guided by the pre-systematic, everyday usages of the notion under consideration. In the present instance, however, it seems to me that the ordinarylanguage analysis of the notion of presumption (or such cognates as 'presumably', 'a presumptive such-and-such') will not get us very far [25, p. 144]”. Instead, she starts her investigation from the technical term 'presumption' as used in legal contexts.

5. See Kauffeld [20] for a discussion of this proposal.

6. He classifies such verbs as "expositives, or expositional performatives" [2, p. 85]. These are explicit performative verbs that show "how the 'statement' is to be fitted into the context of conversation, interlocution, dialogue, or in general of exposition".

7. The notion of positive relevance is defined according to Govier, as follows: "[a] statement A is positively relevant to another statement B if and only if the truth of A counts in favor of the truth of B" [11, p. 148].

8. This contrasts with Hansen's [16] reconstruction of the presumptive inference, on which the inference is a deductively valid one.

9. Kauffeld [18] argues that at least some prima facie counterexamples to the analysis are not real counterexamples. 
10. Godden and Walton [8, p. 323] introduce a distinction between being justified to presume $p$ and being entitled to do so, and suggest that there is an "epistemic sense" of "presumption', and a normative one. However, I have trouble in understanding this distinction. Bermejo-Luque [4, p. 4] and Siegel [p. 1] also point out that the distinction needs to be further developed and clarified. My intuitions do not help at this point, and I sense no distinction between two uses of the ordinary notion.

11. He writes: "Since Godden and Walton provide no reason to suppose that I have misrepresented our ordinary practices, I propose to regard Godden and Walton's criticisms of "Kauffeld's model" as challenges to the capacity of ordinary presuming and presumption to support day-to-argumentation" [19, p. 2].

12. These are the type-B and type-C cases that Kauffeld [19] discusses.

13. Alternatively, it might be the case that my reconstruction of Kauffeld's account as $\mathrm{K} 3$ is incorrect. In that case, I subscribe to the "Kauffeldian" analysis of presumptions in the line of K3. 